\title{
Phantom expansion with non-linear Schrödinger-type formulation of scalar field cosmology
}

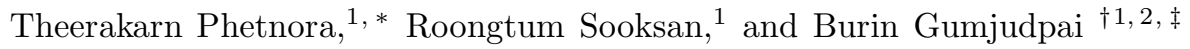 \\ ${ }^{1}$ Fundamental Physics 8 Cosmology Research Unit \\ The Tah Poe Academia Institute (TPTP), Department of Physics \\ Naresuan University, Phitsanulok 65000, Siam \\ ${ }^{2}$ Centre for Theoretical Cosmology, Department of Applied Mathematics and Theoretical Physics \\ University of Cambridge, Centre for Mathematical Sciences \\ Wilberforce Road, Cambridge CB3 OWA, United Kingdom
}

(Dated: October 28, 2018)

\begin{abstract}
We describe non-flat standard Friedmann cosmology of canonical scalar field with barotropic fluid in form of non-linear Schrödinger-type (NLS) formulation in which all cosmological dynamical quantities are expressed in term of Schrödinger quantities as similar to those in time-independent quantum mechanics. We assume the expansion to be superfast, i.e. phantom expansion. We report all Schrödinger-analogous quantities to scalar field cosmology. Effective equation of state coefficient is analyzed and illustrated. We show that in a non-flat universe, there is no fixed $w_{\text {eff }}$ value for the phantom divide. In a non-flat universe, even $w_{\text {eff }}>-1$, the expansion can be phantom. Moreover, in open universe, phantom expansion can happen even with $w_{\text {eff }}>0$. We also report scalar field exact solutions within frameworks of the Friedmann formulation and the NLS formulation in non-flat universe cases.
\end{abstract}

PACS numbers: $98.80 . \mathrm{Cq}$

\section{INTRODUCTION}

Supernovae Type Ia data and cosmic microwave background observations show recently strong evidence of present accelerating phase of the universe [1, 2, 3, 4, 5] while nowadays inflationary paradigm in the early universe is one of the corner stones in cosmology [6]. Present acceleration and inflation of the universe are both believed to result from effect of either dynamical scalar field with time-dependent equation of state coefficient $w_{\phi}(t)<-1 / 3$ or a cosmological constant with $w=-1$. Alternative explanation for present acceleration to dark energy is modification of general relativity which includes braneworld models (for review, see 7] and references therein). Among these ideas, the scalar field catches most attention therefore many analysis in cosmological contexts and observations have been carried out [8]. Conventional formulation of canonical scalar field cosmology with barotropic perfect fluid, can also be expressed as non-linear Ermakov-Pinney equation as shown recently [9, 10]. However, non-Ermakov-Pinney equation for such system can also be written in form of a non-linear Schrödinger-type equation (NLS). The solutions of the NLS-type equation correspond to solutions of the generalized Ermakov-Pinney equation of scalar field cosmology [10, 11]. The NLS-type formulation was concluded and shown in case of power-law expansion in Ref. [12] where all Schrödinger-type quantities corresponding to scalar field cosmology are worked out. NLS-type formulation also provides an alternative way of solving for the scalar field exact solutions in various cases even with non-zero curvature [13].

Various observations allow scalar field equation of state coefficient, $w_{\phi}$ to be less than -1 [15]. Previous evidence from combined cosmic microwave background, large scale structure survey and supernovae type Ia without assuming flat universe yields $w_{\phi}=-1.06_{-0.08}^{+0.13}$ [16] while using supernovae data alone assuming flat universe yields $w_{\phi}=$ $-1.07 \pm 0.09$ [17]. The most recent WMAP five-year result [18, 19] combined with Baryon Acoustic Oscillation (BAO) of large scale structure survey: SDSS and 2dFGRS 20] and type Ia supernovae data from HST [4], SNLS [5] and ESSENCE[17] assuming dynamical $w$ with flat universe yields $-1.33<w_{\phi, 0}<-0.79$ at $95 \%$ confident level [21]. Also this data with additional BBN constraint of limit of expansion rate 22, 23] yields $-1.29<w_{\phi, 0}<-0.79$ at $95 \%$ confident level and $w_{\phi, 0}=-1.04 \pm 0.13$ at $68 \%$ confident level 21]. This suggests that the scalar field could be phantom, i.e $w_{\phi}<-1$ [24]. For a canonical scalar field, phantom behavior can be attained by negative kinetic energy

\footnotetext{
$\dagger$ Corresponding author

*Electronic address: u48313065@nu.ac.th

‡Electronic address: buring@nu.ac.th Electronic address: B.Gumjudpai@damtp.cam.ac.uk
} 
term of the scalar field Lagrangian density. In FLRW general relativistic cosmology, there is a Big Rip singularity with $a, \rho,|p| \rightarrow \infty$ at finite time [25], nevertheless singularity avoidance has been attempted in various ways [26]. Extension to include phantom field case in NLS-type formulation was made in [12]. In NLS-type formulation one can presume any law of expansion $a=a(t)$, e.g. power law $a \sim t^{q}$ or exponential expansion, $a \sim \exp (t / \tau)$ [12, 13 ] and works out all NLS-quantities keeping open possibility for the field to be phantom or non-phantom and non-zero spatial curvature. Analogous studies to the slow-roll, WKB and the Big Rip in NLS formulation were done in [14].

To attain accelerating expansion, one needs to have effective equation of state coefficient, $w_{\text {eff }}<-1 / 3$ where

$$
w_{\text {eff }}=\frac{\rho_{\phi} w_{\phi}+\rho_{\gamma} w_{\gamma}}{\rho_{\text {tot }}},
$$

$\rho_{\gamma}$ is density of barotropic fluid, $\rho_{\phi}$ is density of the scalar field and $\rho_{\text {tot }}=\rho_{\phi}+\rho_{\gamma}$. It has been known in standard cosmology that for flat universe $(k=0)$, if the expansion is $a \sim t^{q}$, then $-1<w_{\text {eff }}<-1 / 3$; if $a \sim \exp (t / \tau)$, then $w_{\text {eff }}=-1$ and if $a \sim\left(t_{\mathrm{a}}-t\right)^{q}$, then $w_{\text {eff }}<-1$. Here $q \equiv 2 /\left[3\left(1+w_{\text {eff }}\right)\right], \tau, t_{\mathrm{a}}$ are finite characteristic times. In the last case, $w_{\text {eff }}<-1$ corresponds to $q<0$.

In this work, we consider phantom expansion $a \sim\left(t_{\mathrm{a}}-t\right)^{q}$ in the NLS-type formulation with non-zero curvature $k$. We introduce cosmological system in Sec. II then NLS-type formulation in Sec. III The Schrödinger quantities for phantom expansion are presented in Sec. [V] where we analyze value of $w_{\text {eff }}$ and show conditions of how much negative $w_{\phi}$ must be in order to keep the expansion phantom. We also illustrate parametric plots for $w_{\text {eff }}$ with $q$ and $t$. Scalar field exact solutions solved from both standard formulation and NLS-type formulation are given in Sec. V where we comment on both procedures of obtaining the solutions. Finally we conclude our work in Sec. VI]

\section{COSMOLOGICAL SYSTEM}

Barotropic fluid and scalar field fluid are major components in our scenario. The perfect barotropic fluid pressure $p_{\gamma}$ and density $\rho_{\gamma}$ obey an equation of state, $p_{\gamma}=(\gamma-1) \rho_{\gamma}=w_{\gamma} \rho_{\gamma}$ while for scalar field, $p_{\phi}=w_{\phi} \rho_{\phi}$. Total density and total pressure are $\rho_{\text {tot }}=\rho_{\gamma}+\rho_{\phi}$ and $p_{\text {tot }}=p_{\gamma}+p_{\phi}$. For the barotropic fluid, $w_{\gamma}$ is written in term of $n$. We set $w_{\gamma} \equiv(n-3) / 3$ so that $n=3\left(1+w_{\gamma}\right)=3 \gamma$, hence $w_{\gamma}=-1$ corresponds to $n=0, w_{\gamma}=-1 / 3$ to $n=2, w_{\gamma}=0$ to $n=3, w_{\gamma}=1 / 3$ to $n=4$, and $w_{\gamma}=1$ to $n=6$. The conservation equation is hence

$$
\dot{\rho}_{\gamma}=-n H \rho_{\gamma}
$$

with solution,

$$
\rho_{\gamma}=\frac{D}{a^{n}}
$$

Therefore $p_{\gamma}=[(n-3) / 3]\left(D / a^{n}\right)$, where $a$ is scale factor, the dot denotes time derivative, $H=\dot{a} / a$ is Hubble parameter and $D \geq 0$ is a proportional constant. We consider scalar field that is minimally coupling to gravity with Lagrangian density, $\mathcal{L}=(1 / 2) \epsilon \dot{\phi}^{2}-V(\phi)$, of which $\epsilon=1$ for non-phantom case and -1 for phantom case. Density and pressure of the field are given as

$$
\rho_{\phi}=\frac{1}{2} \epsilon \dot{\phi}^{2}+V(\phi), \quad p_{\phi}=\frac{1}{2} \epsilon \dot{\phi}^{2}-V(\phi),
$$

therefore

$$
w_{\phi}=\frac{p_{\phi}}{\rho_{\phi}}=\frac{\epsilon \dot{\phi}^{2}-2 V(\phi)}{\epsilon \dot{\phi}^{2}+2 V(\phi)} .
$$

The field obeys conservation equation

$$
\epsilon[\ddot{\phi}+3 H \dot{\phi}]+\frac{\mathrm{d} V}{\mathrm{~d} \phi}=0 .
$$

Considering Friedmann-Lemaître-Robertson-Walker (FLRW) universe, the Friedmann equation and acceleration equation are

$$
\begin{aligned}
H^{2} & =\frac{\kappa^{2}}{3} \rho_{\mathrm{tot}}-\frac{k}{a^{2}}, \\
\frac{\ddot{a}}{a} & =-\frac{\kappa^{2}}{6} \rho_{\mathrm{tot}}\left(1+3 w_{\mathrm{eff}}\right),
\end{aligned}
$$


where $\kappa^{2} \equiv 8 \pi G=1 / M_{\mathrm{P}}^{2}, G$ is Newton's gravitational constant, $M_{\mathrm{P}}$ is reduced Planck mass and $k$ is spatial curvature. Using Eqs. (3), (4), (6) and (7), one can show that

$$
\begin{aligned}
\epsilon \dot{\phi}(t)^{2} & =-\frac{2}{\kappa^{2}}\left[\dot{H}-\frac{k}{a^{2}}\right]-\frac{n D}{3 a^{n}}, \\
V(\phi) & =\frac{3}{\kappa^{2}}\left[H^{2}+\frac{\dot{H}}{3}+\frac{2 k}{3 a^{2}}\right]+\left(\frac{n-6}{6}\right) \frac{D}{a^{n}} .
\end{aligned}
$$

\section{NLS-TYPE FORMULATION}

Non-linear Schrödinger-type formulation for canonical scalar field cosmology and barotropic fluid was proposed by J. D'Ambroise and F. L. Williams [11] and was also extended to include phantom field case $[12]^{1}$. In the Schrödinger formulation, wave function $u(x)$ is related to scale factor in cosmology as

$$
u(x) \equiv a(t)^{-n / 2},
$$

while Schrödinger total energy $E$ and Schrödinger potential $P(x)$ are linked to cosmology as

$$
\begin{aligned}
E & \equiv-\frac{\kappa^{2} n^{2}}{12} D, \\
P(x) & \equiv \frac{\kappa^{2} n}{4} a(t)^{n} \epsilon \dot{\phi}(t)^{2} .
\end{aligned}
$$

These quantities satisfy a non-linear Schrödinger-type equation:

$$
\frac{\mathrm{d}^{2}}{\mathrm{~d} x^{2}} u(x)+[E-P(x)] u(x)=-\frac{n k}{2} u(x)^{(4-n) / n},
$$

with a mapping from $t$ to $x$ is via

$$
x=\sigma(t)
$$

such that $[12,13]$

$$
\begin{aligned}
& \dot{x}(t)=u(x), \\
& \phi(t)=\psi(x)=\frac{ \pm 2}{\kappa \sqrt{n}} \int \sqrt{\frac{P(x)}{\epsilon}} \mathrm{d} x .
\end{aligned}
$$

If $P(x) \neq 0$ and $n \neq 0$, inverse function of $\psi(x)$ exists as $\psi^{-1}(x)$. Therefore $x(t)=\psi^{-1} \circ \phi(t)$ and the scalar field potential, $V \circ \sigma^{-1}(x)$ can be expressed as,

$$
V(t)=\frac{12}{\kappa^{2} n^{2}}\left(\frac{\mathrm{d} u}{\mathrm{~d} x}\right)^{2}-\frac{2 u^{2}}{\kappa^{2} n} P(x)+\frac{12 u^{2}}{\kappa^{2} n^{2}} E+\frac{3 k u^{4 / n}}{\kappa^{2}} .
$$

\section{PHANTOM EXPANSION}

Expansion of the form $a \sim\left(t_{\mathrm{a}}-t\right)^{q}$ is called phantom when $q<0$ for a flat universe. Here in non-flat universe, $q$ is considered to possess any value and the term phantom expansion also refers to expansion function of the form $a \sim\left(t_{\mathrm{a}}-t\right)^{q}$ as in the flat case.

\footnotetext{
${ }^{1}$ It is worth noting that Schrödinger-type equation in scalar field cosmology was previously considered in different procedure to study inflation and phantom field problems [27].
} 


\section{A. NLS-type formulation for phantom expansion}

With the phantom expansion, $a \sim\left(t_{\mathrm{a}}-t\right)^{q}$, we use Eqs. (11) and (16) to relate Schrödinger wave function to standard cosmological quantity as

$$
u(x)=\dot{x}(t)=\left(t_{\mathrm{a}}-t\right)^{-q n / 2} .
$$

Integrate the equation above so that the Schrödinger scale, $x$ is related to cosmic time scale, $t$ as

$$
x(t)=\frac{1}{\beta}\left(t_{\mathrm{a}}-t\right)^{-\beta}+x_{0}
$$

where $\beta \equiv(q n-2) / 2$ and $x_{0}$ is an integrating constant. Conversely,

$$
t(x)=t_{\mathrm{a}}-\frac{1}{\left[\beta\left(x-x_{0}\right)\right]^{1 / \beta}} .
$$

The Schrödinger wave function can be directly found from Eqs. (19) and (21) as

$$
u(x)=\left[\beta\left(x-x_{0}\right)\right]^{q n /(q n-2)} .
$$

For $a \sim\left(t_{\mathrm{a}}-t\right)^{q}$, we can find $\epsilon \dot{\phi}(t)^{2}$ from Eq. (9):

$$
\epsilon \dot{\phi}(t)^{2}=\frac{2 q}{\kappa^{2}\left(t_{\mathrm{a}}-t\right)^{2}}+\frac{2 k}{\kappa^{2}\left(t_{\mathrm{a}}-t\right)^{2 q}}-\frac{n D}{3\left(t_{\mathrm{a}}-t\right)^{q n}} .
$$

Using (23) with phantom expansion in Eq. (13), therefore

$$
P(t)=\frac{q n}{2}\left(t_{\mathrm{a}}-t\right)^{q n-2}+\frac{k n}{2}\left(t_{\mathrm{a}}-t\right)^{q(n-2)}-\frac{\kappa^{2} n^{2} D}{12},
$$

which can be expressed in term of $x$ using Eq. (21) as

$$
P(x)=\frac{2 q n}{(q n-2)^{2}} \frac{1}{\left(x-x_{0}\right)^{2}}+\frac{k n}{2}\left[\frac{2}{(q n-2)\left(x-x_{0}\right)}\right]^{2 q(n-2) /(q n-2)}-\frac{\kappa^{2} n^{2} D}{12} .
$$

One might have a thought that all functions in phantom expansion case can be changed to those in power-law expansion case by interchanging $\left(t_{\mathrm{a}}-t\right) \Leftrightarrow t$. However when $\left(t_{\mathrm{a}}-t\right)$ is differentiated, there is an extra minus sign. The Eq. (25) slightly defers from that of the power-law expansion case because in the power-law case, the numerator of the second term is -2 instead of 2 . The Schrödinger kinetic energy $T$ is negative value of the first two terms of the Schrödinger potential. At last, the scalar field potential obtained from Eq. (18) is

$$
V(t)=\frac{q(3 q-1)}{\kappa^{2}\left(t_{\mathrm{a}}-t\right)^{2}}+\frac{2 k}{\kappa^{2}\left(t_{\mathrm{a}}-t\right)^{2 q}}+\left(\frac{n-6}{6}\right) \frac{D}{\left(t_{\mathrm{a}}-t\right)^{q n}} .
$$

which can be checked by using $a \sim\left(t_{\mathrm{a}}-t\right)^{q}$ in (10). Wave function of the NLS-formulation is found to be nonnormalizable [12] as seen Fig. in 1 for case of phantom expansion with various types of barotropic fluid. Here $q$ is chosen to -6.666 . In flat universe $q=-6.666$ can be attained when $w_{\text {eff }}=-1.1$. Fig. 2 shows $P(x)$ plots for three cases of $k$ with dust and radiation. In there $x_{0}=1$, therefore $P(x)$ goes to negative infinity at $x=1$.

\section{B. Analysis on effective equation of state coefficient}

The definition of effective equation of state coefficient, $w_{\text {eff }}=\left(\rho_{\phi} w_{\phi}+\rho_{\gamma} w_{\gamma}\right) / \rho_{\text {tot }}$ together with Eq. (4) and the results in Eqs. (23) and (26) in context of phantom expansion $a \sim\left(t_{\mathrm{a}}-t\right)^{q}$, we can derive

$$
w_{\mathrm{eff}}=\frac{\left(-3 q^{2}+2 q\right)\left(t_{\mathrm{a}}-t\right)^{2 q-2}-k}{3 q^{2}\left(t_{\mathrm{a}}-t\right)^{2 q-2}+3 k} .
$$




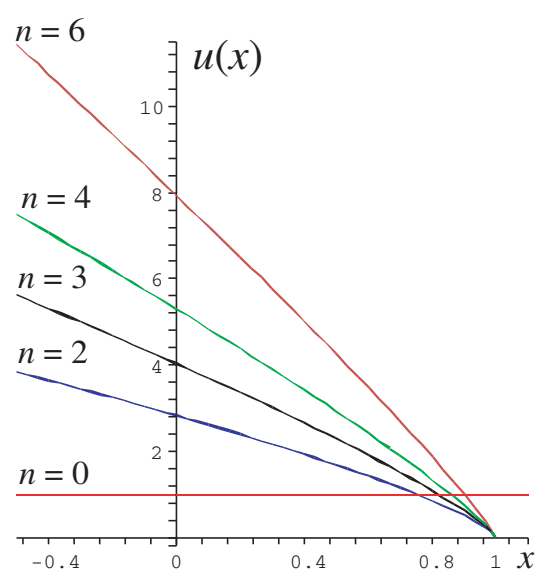

FIG. 1: Schrödinger wave function, $u(x)$ when assuming phantom expansion. $u(x)$ depends on only $q, n$ and $t_{\mathrm{a}}$ but does not depend on $k$. Here we set $t_{\mathrm{a}}=1.0$ and $q=-6.666$. If $k=0, q=-6.666$ corresponds to $w_{\text {eff }}=-1.1$.

There is a locus,

$$
t=t_{\mathrm{a}}-\left(\frac{-k}{q^{2}}\right)^{1 /(2 q-2)}
$$

where $w_{\text {eff }}$ becomes infinite along the locus. Hence for $k=-1$ the locus is $t=t_{\mathrm{a}}-q^{-1 /(q-1)}$ (in term of $x$, it is $\left.x=[2 /(q n-2)] q^{(q n-2) / 2(q-1)}+x_{0}\right)$. Hence for $k=0$, the coefficient $w_{\text {eff }}$ is infinite at $q=0$ or $t=t_{\mathrm{a}}$. It seems from the equation above that $w_{\text {eff }}$ does not depend on properties, $n$ or amount of the barotropic fluid, $D$. Indeed $w_{\text {eff }}$ implicitly depends on $D$ and $n$ since time variable and $q$ are related to $\rho_{\gamma}$ in the Friedmann equation. If $k=0$, it reduces to $q=2 / 3\left(1+w_{\text {eff }}\right)$ and therefore the phantom condition $w_{\text {eff }}<-1$ implies $q<0$ as it is known. This corresponds to a condition,

$$
w_{\phi}<-1-\left(1+w_{\gamma}\right) \frac{\rho_{\gamma}}{\rho_{\phi}} .
$$

Therefore for a fluid with $w_{\gamma}>-1, w_{\phi}$ is always less than -1 in a flat universe. In order to have the expansion $a \sim\left(t_{\mathrm{a}}-t\right)^{q}$ in $k=0$ universe, we must have $w_{\text {eff }}<-1$, i.e. in phantom region. We can rewrite $w_{\phi}$ in term of $w_{\text {eff }}$ as

$$
w_{\phi}=\frac{\left[\frac{3 q^{2}}{\kappa^{2}}\left(t_{\mathrm{a}}-t\right)^{-2}+\frac{3 k}{\kappa^{2}}\left(t_{\mathrm{a}}-t\right)^{-2 q}\right] w_{\mathrm{eff}}-\frac{n-3}{3} D\left(t_{\mathrm{a}}-t\right)^{-q n}}{\frac{3 q^{2}}{\kappa^{2}}\left(t_{\mathrm{a}}-t\right)^{-2}+\frac{3 k}{\kappa^{2}}\left(t_{\mathrm{a}}-t\right)^{-2 q}-D\left(t_{\mathrm{a}}-t\right)^{-q n}} .
$$

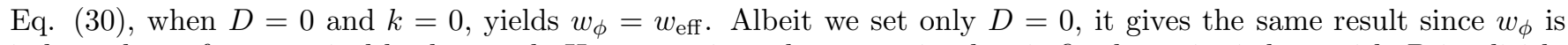
independent of geometrical background. However, since the expansion law is fixed, $w_{\phi}$ is tied up with $D$ implicitly via Eq. (11). Note that $w_{\phi}$ has value in the range $(-\infty,-1]$ and $[1, \infty)$ so that the phantom crossing can not happen when the scalar field is dominant. However, presence of the dust barotropic fluid in the system gives a multiplication factor that is less than 1 to the equation of state, i.e.

$$
w_{\text {eff }}=\left(\frac{\rho_{\phi}}{\rho_{\phi}+\rho_{\gamma}}\right) w_{\phi} .
$$

We can see that the phantom crossing from $w_{\text {eff }}>-1$ to $w_{\text {eff }}<-1$ can happen in this situation. Fig. 3 presents parametric plots of the $w_{\text {eff }}, q, t$ diagram for various $k$ values. From the figure, we see the locus in Eq. (28) where $w_{\text {eff }}$ blows up. In the parametric plots, the value of $w_{\text {eff }}$ at any instance can be obtained if we know the value of $q$. We need to know $q$ from observation in order to know the realistic value of $w_{\text {eff }}$ or the other way around. Fig. 4 plotted from Eq. (27) setting $t_{a}=1$ and $t=0.7$ shows that if $k= \pm 1, q$ could be negative, i.e. phantom accelerated expansion, even when $w_{\text {eff }}>-1$. Regardless of $t_{a}$ and $t$,

$$
\lim _{q \rightarrow-\infty} w_{\mathrm{eff}}(q)=-1 \quad \text { and } \quad \lim _{q \rightarrow+\infty} w_{\mathrm{eff}}(q)=-\frac{1}{3},
$$




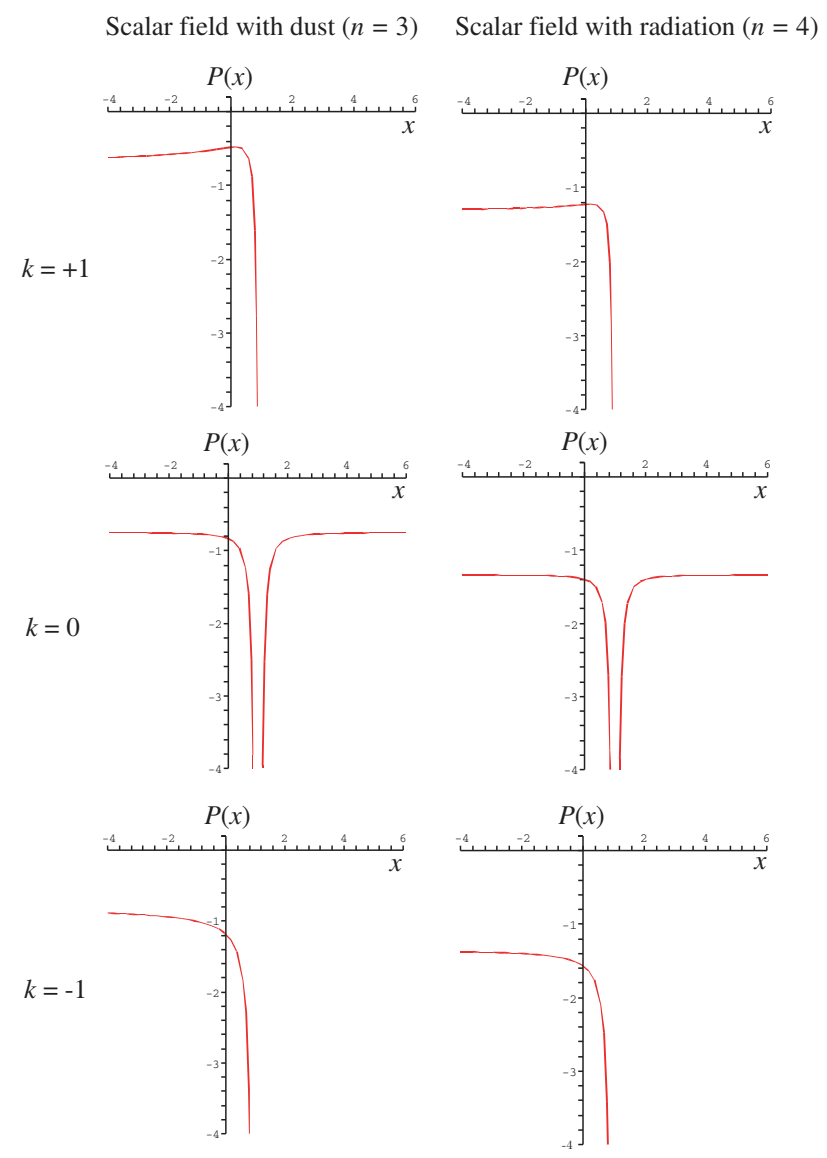

FIG. 2: Schrödinger potential in phantom expansion case for dust and radiation fluids with $k=0, \pm 1$. Numerical parameters are as in the $u(x)$ plots (Fig. 1). $x_{0}$ is set to 1. For non-zero $k$, there is only one real branch of $P(x)$.

for phantom expansion. In particular, for $k=-1, w_{\text {eff }}>0$ could give $q<0$ and $w_{\text {eff }}$ is infinite when $\ln q / \ln \left(t_{\mathrm{a}}-t\right)+$ $q=1$ (see Eq. (28)).

\section{SCALAR FIELD EXACT SOLUTION}

\section{A. Bound value of $\phi(t)$ from effective equation of state for $k=0$ case}

In flat universe, the phantom expansion occurs when $w_{\text {eff }}<-1$. Using Eqs. (4), (5) in Eq. (1), we get a bound

$$
\epsilon \dot{\phi}^{2}<-\frac{n}{3} \rho_{\gamma} .
$$

Assuming $a(t)=\left(t_{\mathrm{a}}-t\right)^{q}$ and phantom scalar field, i.e. $\epsilon=-1$ with using Eq. (3), the solution is found to be in the region,

$$
\phi(t)>\frac{1}{\beta} \sqrt{\frac{D n}{3}}\left(t_{\mathrm{a}}-t\right)^{-\beta}+\phi_{0}
$$

where $\beta \equiv(q n-2) / 2$. 

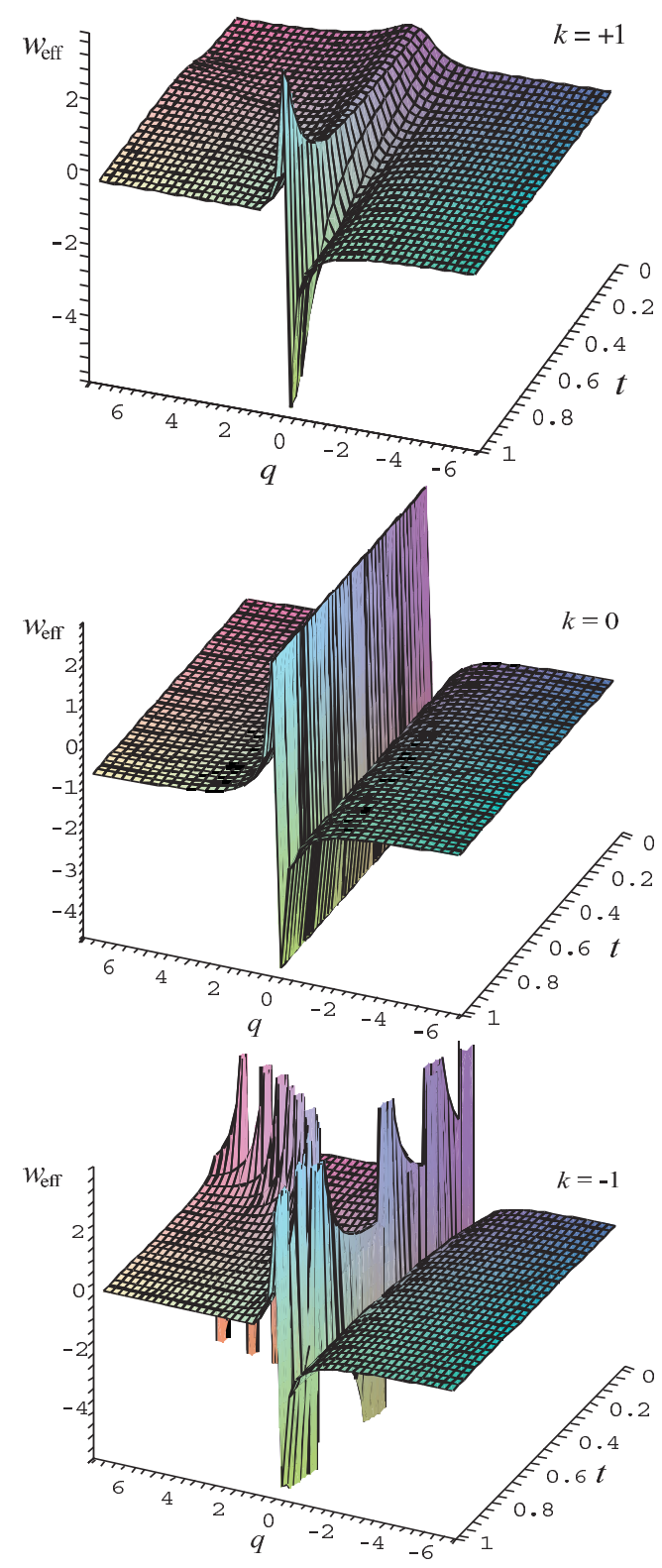

FIG. 3: Parametric plots of $w_{\text {eff }}$ for the expansion $a \sim\left(t_{\mathrm{a}}-t\right)^{q}$ in closed, flat and open universe. Here $t_{\mathrm{a}}$ is set to 1 .

\section{B. Solution solved from Friedmann equation}

\section{Scalar field potential in flat and scalar field dominated case}

A simplest case for analysis is when considering flat universe $(k=0)$ with negligible amount of barotropic fluid $(D=0)$. The Eq. (23) is hence simply integrated out. The solution is

$$
\phi(t)= \pm \frac{1}{\kappa} \sqrt{\frac{2 q}{\epsilon}} \ln \left(t_{\mathrm{a}}-t\right)+\phi_{0}
$$

Insert this result into Eq. (26), we obtain the scalar field potential,

$$
V(\phi)=\frac{q(3 q-1)}{\kappa^{2}} \exp \left\{ \pm \kappa \sqrt{\frac{2 \epsilon}{q}}\left[\phi(t)-\phi_{0}\right]\right\}
$$




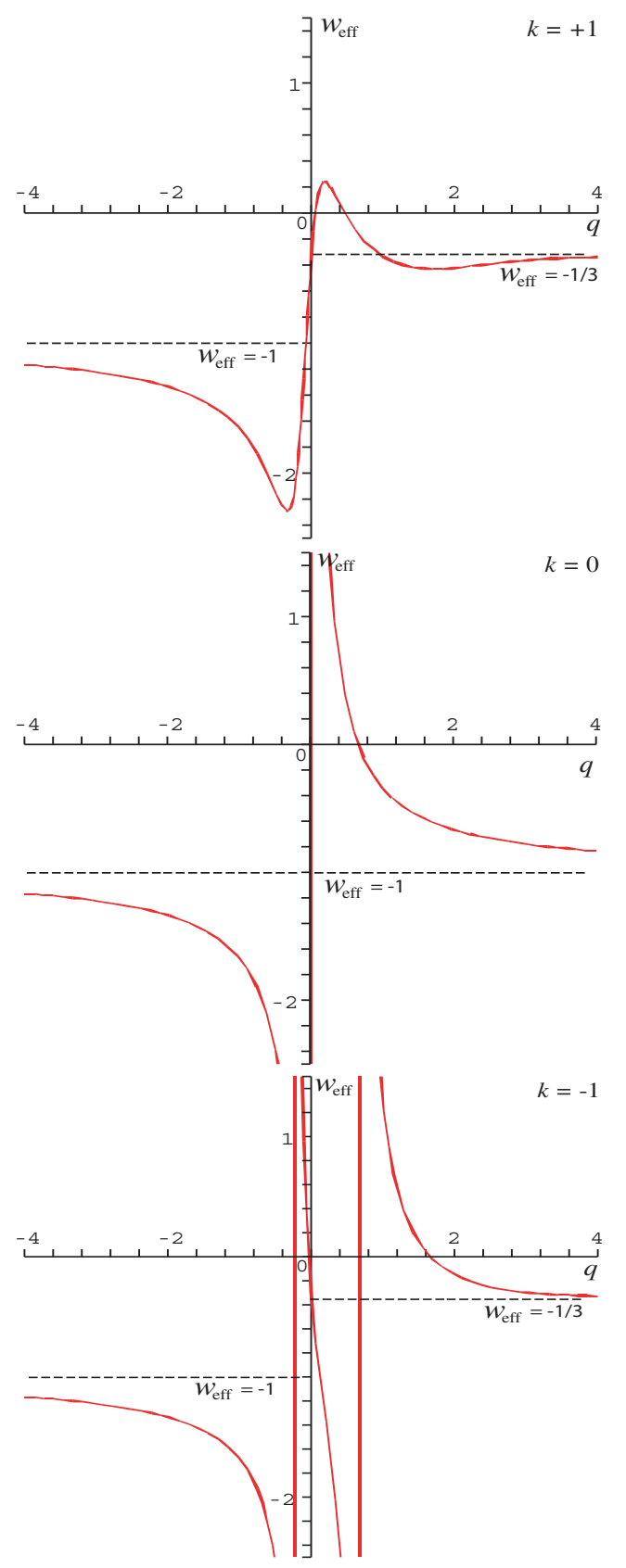

FIG. 4: $w_{\text {eff }}$ for the expansion $a \sim\left(t_{\mathrm{a}}-t\right)^{q}$ in closed, flat and open universe. Here $t_{\mathrm{a}}$ is set to 1 and $t$ is 0.7 .

The solutions above are real only when $q$ and $\epsilon$ have the same sign, i.e. when $\epsilon=1, q>0$ and $\epsilon=-1, q<0$. This looks similar to potential that gives power-law expansion as well-known [28]. It is not surprised since in our case $(q<0)$ it has been known that phantom field, when rolling up the hill of slope-varying exponential potential (varying $q$ ), results in phantom expansion $a \sim\left(t_{\mathrm{a}}-t\right)^{q}[25]$. 
2. Solution for $k=0, D \neq 0$ case

For the case $k=0$ with $D \neq 0$, the solution of Eq. (23) is

$$
\begin{aligned}
\phi(t)= & \pm \frac{1}{q n-2} \sqrt{\frac{2 q}{\epsilon \kappa^{2}}} \times \\
& \left\{\ln \left[\frac{\left(t_{\mathrm{a}}-t\right)^{-q n+2}}{\left(1+\sqrt{1-\left(n D \kappa^{2} / 6 q\right)\left(t_{\mathrm{a}}-t\right)^{-q n+2}}\right)^{2}}\right]+2 \sqrt{1-\frac{n D \kappa^{2}\left(t_{\mathrm{a}}-t\right)^{-q n+2}}{6 q}}+\ln \left(\frac{-n D \kappa^{2}}{6 q}\right)\right\}+\phi_{0},
\end{aligned}
$$

which is infinite when $q=2 / n$. The last logarithmic term in the bracket is an integrating constant. Logarithmic function is valid only when $q<0$.

\section{Solution for $k \neq 0, D=0$ case}

For the reverse case, $k \neq 0, D=0$, the solution is

$$
\begin{aligned}
\phi(t)= & \pm \frac{1}{q-1} \sqrt{\frac{2 q}{\epsilon \kappa^{2}}} \times \\
& \left\{\ln \left[\frac{\left(t_{\mathrm{a}}-t\right)^{q-1}}{\sqrt{k / q}}\left(1+\sqrt{\left(\frac{k}{q}\right)\left(t_{\mathrm{a}}-t\right)^{-2 q+2}+1}\right)\right]-\sqrt{\left(\frac{k}{q}\right)\left(t_{\mathrm{a}}-t\right)^{-2 q+2}+1}\right\}+\phi_{0},
\end{aligned}
$$

which becomes infinite when $q=1$. The values $q$ and $\epsilon$ must have the same sign for it to be real-value function. The case $k \neq 0$ with $D \neq 0$ can not be found analytically except when setting $n=2\left(w_{\gamma}=-1 / 3\right)$ which is not natural fluid.

\section{Solution solved with NLS-type formulation}

One can obtain exact solution of Eq. (23) indirectly via NLS-type formulation. Consider Eq. (25), we notice that setting $D=0$ does not make sense in NLS-formulation since even $D$ vanishes, $n$ (barotropic fluid parameter) still appears in other terms. Therefore we can only consider non-zero $D$ case. Assuming $k=0$ with $D \neq 0$ and using Eq. (25) in Eq. (17), the solution is

$$
\begin{aligned}
\psi(x)= & \pm \sqrt{\frac{8 q}{\epsilon \kappa^{2}(q n-2)^{2}} \times} \\
& \left\{-\sqrt{1-\left[\frac{\kappa^{2} D n(q n-2)^{2}}{24 q}\left(x-x_{0}\right)^{2}\right]}+\ln \left[\frac{1+\sqrt{1-\left[\kappa^{2} D n(q n-2)^{2} / 24 q\right]\left(x-x_{0}\right)^{2}}}{\left(x-x_{0}\right)} \frac{4 q n}{\epsilon(q n-2)^{2}}\right]\right\} .
\end{aligned}
$$

Transforming to $t$ variable using Eq. (20),

$$
\begin{aligned}
\phi(t)= & \pm \frac{1}{q n-2} \sqrt{\frac{2 q}{\epsilon \kappa^{2}}} \times \\
& \left\{\ln \left[\frac{\left(t_{\mathrm{a}}-t\right)^{-q n+2}}{\left(1+\sqrt{1-\left(n D \kappa^{2} / 6 q\right)\left(t_{\mathrm{a}}-t\right)^{-q n+2}}\right)^{2}}\right]+2 \sqrt{1-\frac{n D \kappa^{2}\left(t_{\mathrm{a}}-t\right)^{-q n+2}}{6 q}}+\ln \left(\frac{q n-2}{2 q n}\right)^{2}\right\}+\phi_{0} .
\end{aligned}
$$

The only difference from the solution (37) obtained from standard method is the logarithmic integrating constant term in the bracket. In case of $k \neq 0$ with $D \neq 0$, the integral (17) can not be integrated analytically even when assuming $n$ value except for $n=2$ which is integrable. However $n=2$ is not natural fluid. This is similar to using standard method in Sec. VB3. 


\section{CONCLUSIONS}

We consider a system of FLRW cosmology of scalar field and barotropic fluid assuming phantom acceleration. We have worked out cosmological quantities in the NLS-formulation of the system for flat and non-flat curvature. The Schrödinger wave functions are illustrated in Fig. 1 for various types of barotropic fluid. These wave functions are non-normalizable. We show Schrödinger potential plots for dust and radiation cases in closed, flat and open universe. The procedure considered here is reverse to a problem solving in quantum mechanics in which the Schrödinger potential must be known before solving for wave function. In NLS formulation, the Schrödinger equation is non-linear (reducible to linear in some cases) and the wave function is expressed first by the expansion function, $a(t)$. Afterward the Schrödinger potential is worked out based on expansion function assumed. Moreover, the NLS total energy $E$ is negative (see Eq. (12)). We also perform analysis on effective equation of state. We expresses $w_{\text {eff }}$ in term of $q$ and

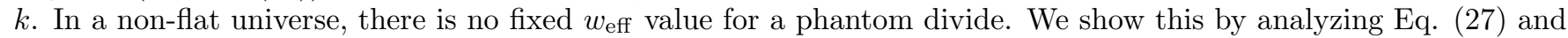
by presenting illustrations in Figs. (3) and (4). In these plots, even $w_{\text {eff }}>-1$, the expansion can still be phantom, i.e. $q$ can be negative. Especially, in $k=-1$ case, positive $w_{\text {eff }}$ could also give $q<0$. The value of $w_{\text {eff }}$ approaches -1 when $q \rightarrow-\infty$ and $-1 / 3$ when $q \rightarrow+\infty$. In open universe, $w_{\text {eff }}$ blows up when $\ln q / \ln \left(t_{\mathrm{a}}-t\right)+q=1$.

The last part of this work is to solve for scalar field exact solution for phantom expansion. Within framework of the standard Friedmann formulation, we obtained exact solution in simplest case where scalar field is dominated in flat universe. Apart from that we also obtained exact solutions in the cases of non-flat universe with scalar field domination and flat universe with mixture of barotropic fluid and scalar field. Afterward, we use NLS formulation, in which the wave function is equivalent to the scalar field exact solution, to solve for the exact solutions. We can apply the NLS method to solve for the solution only when the barotropic fluid density is non-negligible. Setting $D=0$ in NLS framework is not sensible because even if $D$ term vanishes, the barotropic fluid parameter $n$ still appears in other terms of the wave function. This is a disadvantage point of the NLS formulation.

Transforming standard Friedmann formulation to NLS formulation renders a few effects to the integration. In standard form (Eq. (23) $), n$ appears in only $D$-term and all terms are $t$-dependent. In NLS-form (Eq. (25) when inserted in Eq. (17)), $D$-term becomes a constant $(E)$, hence the number of $x$ (or equivalently $t$ )-dependent terms is reduced by one. This is a good aspect of the NLS. In both Friedmann-form and NLS-form, the solutions when $k \neq 0$ and $D \neq 0$ are difficult or might be impossible to integrate unless assuming values of $q$ and $n$. Therefore reduction number of $x$-dependent term helps simplifying the integration.

\section{Acknowledgements}

T. P. is sponsored by the Thailand Riean Dee Science Studentship. R. S. is supported via the Tah Poe Academia Institute's Research Assistantship under the Naresuan Faculty of Science Research Scheme. B. G. is a TRF Research Scholar under the Thailand Research Fund. B. G. expresses his gratitude to Naresuan University's Overseas Visiting Postdoctoral Research Fellowship and to Anne-Christine Davis, Stephen Hawking and Neil Turok for providing additional support at the Centre for Theoretical Cosmology, D.A.M.T.P., University of Cambridge.

[1] S. Masi et al., Prog. Part. Nucl. Phys. 48 (2002) 243; C. L. Bennett et al., Astrophys. J. Suppl. 148 (2003) 1; WMAP Collab. (D. N. Spergel et al.), Astrophys. J. Suppl. 148 (2003) 175.

[2] SDSS Collab. (R. Scranton et al.), arXiv: astro-ph/0307335.

[3] Supernova Search Team Collab. (A. G. Riess et al.), Astron. J. 116 (1998) 1009; Supernova Cosmology Project Collab. (S. Perlmutter et al.), Astrophys. J. 517 (1999) 565; A. G. Riess, arXiv: astro-ph/9908237; The Supernova Cosmology Project Collab. (G. Goldhaber et al.), arXiv: astro-ph/0104382; Supernova Search Team Collab. (J. L. Tonry et al.), Astrophys. J. $\mathbf{5 9 4}$ (2003) 1.

[4] Supernova Search Team Collab. (A. G. Riess et al.), Astrophys. J. 607 (2004) 665; A. G. Riess et al., Astrophys. J. 659 (2007) 98.

[5] SNLS Collab. (P. Astier et al.), Astron. Astrophys. 447 (2006) 31.

[6] D. Kazanas, Astrophys. J. 241 (1980) L59; A. A. Starobinsky, Phys. Lett. B 91 (1980) 99; A. H. Guth, Phys. Rev. D 23 (1981) 347; K. Sato, Mon. Not. Roy. Astro. Soc. 195 (1981) 467; A. Albrecht and P. J. Steinhardt, Phys. Rev. Lett. 48 (1982) 1220; A. D. Linde, Phys. Lett. B 108 (1982) 389.

[7] S. Nojiri and S. D. Odintsov, Int. J. Geom. Meth. Mod. Phys. 4 (2007) 115.

[8] T. Padmanabhan, Curr. Sci. 88 (2005) 1057; E. J. Copeland, M. Sami and S. Tsujikawa, Int. J. Mod. Phys. D 15 (2006) 1753; T. Padmanabhan, Dark energy: mystery of the millennium, in AIP Conf. Proc. Albert Einstein's Century Int. Conf., eds. J.-M. Alimi and A. Füzfa (AIP, New York, 2006), 861 p. 179. 
[9] R. M. Hawkins and J. E. Lidsey, Phys. Rev. D 66 (2002) 023523; F. L. Williams and P. G. Kevrekidis, Class. Quant. Grav. 20 (2003) L177; J. E. Lidsey, Class. Quant. Grav. 21 (2004) 777; F. L. Williams, P. G. Kevrekidis, T. Christodoulakis, C. Helias, G. O. Papadopoulos and T. Grammenos, On 3+1 dimensional scalar field cosmologies, in Trends in Gen. Rel. and Quant. Cosmol., (Nova Science Pub., 2006) p. 37; A. Kamenshchik, M. Luzzi and G. Venturi, arXiv: math-ph/0506017.

[10] F. L. Williams, Int. J. Mod. Phys. A 20 (2005) 2481.

[11] J. D'Ambroise and F. L. Williams, Int. J. Pure Appl. Maths. 34 (2007) 117.

[12] B. Gumjudpai, Astropart. Phys. 30 (2008) 186.

[13] B. Gumjudpai, Gen. Rel. Grav. 41 (2009) 249.

[14] B. Gumjudpai, J. Cosmol. Astropart. Phys. 0809 (2008) 028.

[15] A. Melchiorri, L. Mersini-Houghton, C. J. Odman and M. Trodden, Phys. Rev. D 68 (2003) 043509; P. S. Corasaniti, M. Kunz, D. Parkinson, E. J. Copeland and B. A. Bassett, Phys. Rev. D 70 (2004) 083006; U. Alam, V. Sahni, T. D. Saini and A. A. Starobinsky, Mon. Not. Roy. Astron. Soc. 354 (2004) 275.

[16] WMAP Collab. (D. N. Spergel et al. ), Astrophys. J. Suppl. 170 (2007) 377.

[17] ESSENCE Collab. (W. M. Wood-Vasey et al.), Astrophys. J. 666 (2007) 694.

[18] WMAP Collab. (G. Hinshaw et al.), arXiv:0803.0732 [astro-ph].

[19] WMAP Collab. (J. Dunkley et al.), arXiv:0803.0586 [astro-ph].

[20] W. J. Percival, S. Cole, D. J. Eisenstein, R. C. Nichol, J. A. Peacock, A. C. Pope and A. S. Szalay, Mon. Not. Roy. Astron. Soc. 381 (2007) 1053.

[21] WMAP Collab. (E. Komatsu et al.), arXiv:0803.0547 [astro-ph] (version 2).

[22] G. Steigman, Ann. Rev. Nucl. Part. Sci. 57 (2007) 463.

[23] E. L. Wright, Astrophys. J. 664 (2007) 633.

[24] R. R. Caldwell, Phys. Lett. B 545 (2002) 23; G. W. Gibbons, arXiv:hep-th/0302199 S. Nojiri and S. D. Odintsov, Phys. Lett. B $\mathbf{5 6 2}(2003) 147$.

[25] A. A. Starobinsky, Grav. Cosmol. 6 (2000) 157; R. R. Caldwell, M. Kamionkowski and N. N. Weinberg, Phys. Rev. Lett. 91 (2003) 071301; S. Nesseris and L. Perivolaropoulos, Phys. Rev. D 70 (2004) 123529; J. g. Hao and X. z. Li, Phys. Rev. D 67 (2003) 107303; X. z. Li and J. g. Hao, Phys. Rev. D 69 (2004) 107303; P. Singh, M. Sami and N. Dadhich, Phys. Rev. D 68 (2003) 023522; J. G. Hao and X. z. Li, Phys. Rev. D 70 (2004) 043529; M. Sami and A. Toporensky, Mod. Phys. Lett. A 19 (2004) 1509; S. Nojiri, S. D. Odintsov and S. Tsujikawa, Phys. Rev. D 71 (2005) 063004; B. Gumjudpai, T. Naskar, M. Sami and S. Tsujikawa, J. Cosmol. Astropart. Phys. 0506 (2005) 007; L. A. Urena-Lopez, J. Cosmol. Astropart. Phys. 0509 (2005) 013; G. Cognola, E. Elizalde, S. Nojiri, S. Odintsov and S. Zerbini, Phys. Rev. D 73 (2006) 084007; G. Cognola, E. Elizalde, S. Nojiri, S. Odintsov and S. Zerbini, Phys. Rev. D 75 (2007) 086002.

[26] S. Nojiri, S. D. Odintsov and M. Sasaki, Phys. Rev. D 71 (2005) 123509; M. Sami, A. Toporensky, P. V. Tretjakov and S. Tsujikawa, Phys. Lett. B 619 (2005) 193; G. Calcagni, S. Tsujikawa and M. Sami, Class. Quant. Grav. 22 (2005) 3977; H. Wei and R. G. Cai, Phys. Rev. D 72 (2005) 123507; B. M. Leith and I. P. Neupane, J. Cosmol. Astropart. Phys. 0705 (2007) 019; T. Koivisto and D. F. Mota, Phys. Lett. B 644 (2007) 104; T. Koivisto and D. F. Mota, Phys. Rev. D 75 (2007) 023518; D. Samart and B. Gumjudpai, Phys. Rev. D 76 (2007) 043514; T. Naskar and J. Ward, Phys. Rev. D 76 (2007) 063514; B. Gumjudpai, Thai J. Phys. Series 3: Proc. of the SIAM Phys. Cong. 2007 arXiv:0706.3467 [gr-qc]].

[27] S. V. Chervon and V. M. Zhuravlev, arXiv: gr-qc/9907051 A. V. Yurov, arXiv: astro-ph/0305019 A. A. Andrianov, F. Cannata and A. Kamenshchik, Phys. Rev. D 72 (2005) 043531; A. Das, N. Tariq and R. W. M. Woodside, arXiv:gr-qc/0610097, A. V. Yurov, A. V. Astashenok and V. A. Yurov, arXiv: astro-ph/0701597.

[28] F. Lucchin and S. Matarrese, Phys. Rev. D 32 (1985) 1316. 